\title{
Effect of Goal-directed Fluid Therapy on the Shedding of the Glycocalyx Layer in Retroperitoneal Tumour Resection
}

\author{
Xiaoyu Wang ${ }^{1}$, Yi Duan ${ }^{1}$, Zhifeng Gao ${ }^{1}$ and Jie $\mathrm{Gu}^{2}$ \\ ${ }^{1}$ Department of Anaesthesiology, Beijing Tsinghua Changgung Hospital, School of Clinical Medicine, Tsinghua University, Beijing, China \\ ${ }^{2}$ Department of Anaesthesiology, Peking University International Hospital, Beijing, China
}

\begin{abstract}
Objective: To evaluate the effects of different stroke volume variation (SVV) targets on endothelial glycocalyx (EG) shedding. Study Design: Randomised controlled trial.

Place and Duration of Study: Department of Anaesthesiology, Peking University International Hospital, Beijing, China, from February to June 2018.

Methodology: Patients undergoing elective retroperitoneal tumour resection were assigned to one of two groups. SVV $9 \%$ group $(n=40)$, who received a more traditional (liberal) fluid therapy with SVV $\leq 9 \%$; and the SVV $14 \%$ group ( $n=40$ ), received restrictive fluid therapy with SVV $\leq 14 \%$. Patients' serum concentrations of syndecan-1 (SDC-1), interleukin-6 (IL-6) and tumour necrosis factor- $\alpha$ (TNF- $\alpha$ ) were measured at the time that patients entered the operating theatre; at 1-and 4-hours following initiation of the surgery; and at 24- and 72-hours post-surgery. Postoperative complications, and one-year survival rates were also noted.

Results: Perioperative serum SDC-1, IL-6 and TNF- $\alpha$ concentrations were significantly elevated in both groups, which were more pronounced in SVV 9\% ( $p=0.028,<0.001$, and $<0.001$ respectively). A positive correlation was observed between SDC-1 and TNF- $\alpha$, L-6, and infusion volume, respectively. Postoperative complications, including fever and blood transfusion, were more aggravated in SVV 9\% than those in SVV 14\% (80\% vs. 59\%, and 45\% vs. 23.1\%, both p <0.05). However, no significant difference in the one-year survival rate was observed.

Conclusion: Using SVV $14 \%$ as a target for guiding fluid therapy in elective retroperitoneal tumour resection was associated with reduced perioperative glycocalyx shedding, ameliorated inflammatory response, and reduced postoperative complications.
\end{abstract}

Key Words: Randomised controlled trial, Retroperitoneal neoplasms, Glycocalyx, Tumour necrosis factor-alpha, Interleukin-6, Fluid therapy, Stroke volume.

How to cite this article: Wang X, Duan Y, Gao Z, Gu J. Effect of Goal-directed Fluid Therapy on the Shedding of the Glycocalyx Layer in Retroperitoneal Tumour Resection. J Coll Physicians Surg Pak 2021; 31(10):1179-1185.

\section{INTRODUCTION}

Theendothelial glycocalyx (EG) is a negatively-charged, carbohydrate-mesh covering the luminal side of blood vessels. ${ }^{1,2}$ It is crucial in maintaining vascular wall homeostasis and physiological functions of the microcirculation, including vascular permeability, immunity, blood coagulation, and interaction with serum proteins. ${ }^{2,3}$ Unfortunately, the EG is particularly vulnerable to damage in response to sepsis, ischemia-reperfusion, trauma, and surgeries. ${ }^{2,4-6}$ Retroperitoneal tumour (RPT) is a general term for latent retroperitoneal tumours.

Correspondence to: Zhifeng Gao, Department of Anaesthesiology, Beijing Tsinghua Changgung Hospital, School of Clinical Medicine, Tsinghua University, No. 168 Litang Road, Beijing, China

E-mail: sunshine_duan@163.com

Received: October 27, 2020; Revised: August 29, 2021;

Accepted: September 22, 2021

DOI: https://doi.org/10.29271/jcpsp.2021.10.1179
Resection of such tumours characterised by considerable trauma, extended operation procedural times, and significant changes in effective circulating blood volume, often involves profound ED shedding. Thus, prevention of glycocalyx damage may be a promising therapeutic target in patients receiving retroperitoneal tumour resection.

Fluid infusion strategy profoundly impacts on the glycocalyx shedding ${ }^{1,2}$ Hypervolemia is strongly correlated with enhanced glycocalyx degradation. Goal-directed fluid therapy (GDFT) is now recognised as an effective perioperative fluid management. ${ }^{7,8}$ Stroke volume variation (SVV), an indicator of GDFT, may serve as a promising predictor of intraoperative fluid responsiveness during resection of retroperitoneal tumour. ${ }^{8,9}$ Using SVV as a monitoring index, it may more precisely determine the actual effective blood volume of patients during the surgery. SVV < $9 \%$ fluid challenge cannot improve the cardiac output (CO), while SVV >14\% fluid challenge can improve the CO. ${ }^{10}$ However, its range within the bracket of $9 \%$ to $14 \%$ is the grey zone of GDFT. To the best of authors' knowledge, there are 
no reports on the effects of different SVV targets on EG shedding during resection of retroperitoneal tumour. It was hypothesised that a more restrictive fluid therapy with SVV 14\% may lead to reduced perioperative glycocalyx shedding, ameliorated inflammatory response, and improved postoperative complications.

In this study, the objective was to evaluate the effects of different SVV targets on perioperative EG shedding during retroperitoneal tumour resection.

\section{METHODOLOGY}

This is a single-centre, prospective, and randomised controlled clinical study, registered in the China Clinical Research Registry (Registration No. ChiCTR-IIR-17014180). Ethical approval for this study was provided by the Ethical Committee of Peking University International Hospital, Beijing, China (Approval No. 2016-029). The study was conducted at Peking University International Hospital, Beijing, China, from February to June 2018. All subjects were given an informed consent form before participating, which was signed after the subjects were notified of the research study and its intent.

Eligibility criteria were: Diagnosis of retroperitoneal tumours by abdominal CT before surgery, age of 18-65 years, an ASA grade of I-II. ${ }^{11}$ Exclusion criteria were the following: Evidence of pre-existing cardiopulmonary disease, liver and/or kidney dysfunction, systemic infection, abnormal coagulation, hypoproteinaemia, and anaemia seen in any of the prospective study subjects, Surgery that was not completed for various reasons, an operation time that was less than four hours, and patients that simply refused to cooperate midway through the study, resulting in the aggregation of an incomplete data set.

To estimate the sample size, a pilot study was conducted to measure serum concentrations of SDC-1 at four hours following skin incision in 20 patients that received RPT resection (10 in the SVV 9\% group and 10 in the SVV 14\% group). The mean value and standard deviation of both groups were 24.87 \pm 8.94 and $15.28 \pm 6.01$, respectively. With an alpha value of $\alpha=0.05$, two-tailed analysis and the beta value set at $\beta=0.20$, the authors calculated the required sample size to be about 76 cases from this power analysis. Additionally, considering a loss rate of five percent, we enrolled 80 patients, scheduled for retroperitoneal tumour resection.

Computer randomisation of study identification (ID) numbers for both groups was followed by the placement of printed ID number slips into numerically ordered envelopes matching the computer randomisation file. According to the envelope method, the enrolled patients were randomly divided into the SVV $14 \%$ group $(n=40)$ and the SVV9\% group $(n=40)$. After the subject signed the informed consent form, the investigator opened the next sealed, opaque envelope in the numerical sequence to assign the patient to their group. Using the singleblinded principle, subjects were unaware of the grouping.
All patients were anesthetised according to the standard protocol in this hospital. During the operation, lactated Ringer's solution (LR); (Otsuka Pharmaceutical Co., Ltd, China) was continuously infused at a rate of $2-3 \mathrm{ml} / \mathrm{Kg} / / \mathrm{h}$ to maintain intraoperative basic fluid intake (physiological requirement plus evaporation), and the corresponding dose of LR was supplemented according to the urine output volume every 30 minutes. Fluid challenge was performed while monitoring with the aid of the FloTrac/Vigileo system (Edwards Life sciences LLC, US). In the SVV 14\% group, the SVV target value was set to 14 percent. If SVV $>14 \%$, LR was rapidly administered at a volume of $200 \mathrm{ml}$, and reappraise it five minutes later. If SVV was still $\geq 14 \%$, LR was rapidly administered again at a volume of $200 \mathrm{ml}$. If not, the rapid rehydration was suspended. In the same way, the SVV target value was set to 9 percent in the SVV 9\% group. If SVV $\geq 9 \%$, LR was rapidly administered at a volume of $200 \mathrm{ml}$, followed by re-evaluation five minutes later. If SVV remained $\geq 9$ percent, LR was rapidly administered again at a volume of 200 $\mathrm{ml}$. If not, the rapid rehydration was suspended. Meanwhile, cardiac index $(\mathrm{Cl})$ and mean arterial pressure (MAP) were also monitored. If $\mathrm{Cl}<2.5 \mathrm{~L} / \mathrm{min} / / \mathrm{m}$, dopamine was administered at a dose of $5 \mathrm{ug} / \mathrm{Kg} / / \mathrm{min}$ until $\mathrm{Cl} \geq 2.5 \mathrm{~L} / \mathrm{min} / / \mathrm{m}$ was achieved. In case of $\mathrm{Cl} \geq 2.5 \mathrm{~L} / \mathrm{min} / / \mathrm{m}$ and MAP $<65 \mathrm{mmHg}$, norepinephrine was administered at a dose of $0.5 \mathrm{ug} / \mathrm{Kg} / \mathrm{min}$ to achieve a MAP $\geq 65 \mathrm{mmHg}$. Consideration should also be given in the context of blood transfusions (i.e., a red cell suspension and plasma ratio of $1: 1$ ) when the bleeding volume exceeded $1 / 4$ of the blood volume or the haematocrit (HCT) $<25 \%$.

The primary outcome measure was patients' serum concentrations of syndecan-1 (SDC-1); (Perlong Medical Equipment Co., Ltd), which was detected by standard enzyme-linked immunosorbent assay (ELISA); (Perlong Medical Equipment Co., Ltd,) at the following time points: the time that patients entered the operating theatre (T0); at 1 (T1) and 4 (T2) hours following initiation of the surgery; and at 24 (T3) and 72 (T4) hours post-surgery. Secondary outcome measures included: Perioperative changes in serum levels of IL- 6 and TNF- $\alpha$, extubation time, duration of hospital stay, postoperative complications and oneyear survival rate, hemodynamic parameters (i.e., MAP, HR, central venous pressure (CVP), SVV and $\mathrm{Cl}$ ) that were measured every 30 minutes from the time point of skin incision to surgery within 4 hours, the intraoperative fluid volume, urine volume, blood loss, blood transfusion volume, norepinephrine dosage. Postoperative complications, according to the Dindo-Clavien grading system ${ }^{12}$ were all recorded. Patients were followed up for their survival for one year via telephone followup every month.

Data were analysed using the SPSS version 23.0 software programme (IBM). Measured data that followed a normal distribution were expressed as $\bar{x} \pm \mathrm{SD}$, and independent sample Student's $t$-test was used for inter-group comparison. Data collected at each time-point were analysed by repeated measures analysis of variance (ANOVA). The data that did not follow a normal distribution were expressed as the median (and inter-quartile range (IQR), and the Mann-Whitney $U$-test was 
used for inter-group comparisons. Count data were expressed as frequencies or percentages for categorical variables, and Chi-square or Fisher's Exact test was used for inter-group comparison. Pearson correlation analysis was applied in this study and multiple linear regression analysis was used to get its relationship with multiple variables. The Kaplan-Meier method estimated the probability of survival; and log rank test was used for survival analysis. An alpha value of $p \square 0.05$ indicated that the differences between sets of data were statistically significant.

In addition, the authors took T0 as the starting point and T3 as the end-point, and subsequently calculated the concentrations of SDC-1, IL- 6 , and TNF- $\alpha$, urine volume, blood loss, infusion volume, erythrocyte transfusion, plasma transfusion, and norepinephrine dosage infusion volume that were generated during this period, and expressed those values as C-SDC-1, C-IL-6, CTNF- $\alpha$, etc. The authors analysed the correlations between $C$ IL-6, C-TNF- $\alpha$, age, gender, height, weight, body mass index (BMI), C-urine volume, C-blood loss, C-infusion volume, C-erythrocyte transfusion, C-plasma transfusion, C-norepinephrine dosage and C-SDC-1 respectively. First, the authors plotted a scatter plot and performed linear correlation analysis on linearly related variables. Then, multiple linear regression analysis was used to explore linear dependency of the influencing factors on the shedding products of the glycocalyx. All variables had a tolerance of $>0.1$ and a variance inflation factor (VIF) $<10$.

\section{RESULTS}

A total of 80 patients, who were undergone retroperitoneal tumour resection, were enrolled. One patient in the SVV 14\% group refused to participate in the experiment before anaesthesia induction, and was subsequently excluded from the analysis. As a result, there were 40 patients in the SVV $9 \%$ group and 39 in the SVV 14\% group (Figure 1). No unintended effects or harms happened during this study.

There were no significant differences observed in terms of age, body mass index (BMI), gender composition, ASA grade, co-morbidities (i.e., hypertension, diabetes) and surgical duration (all $p$ $>0.05$; Table I).

$\mathrm{HR}, \mathrm{MAP}, \mathrm{CVP}$ and $\mathrm{Cl}$ in both groups changed over time ( $\mathrm{p}$ $<0.001$ ), and compared with the SVV 9\% group. It was found that CVP decreased in the SVV $14 \%$ group ( $p<0.05)$. No difference in HR, MAP and Cl was observed between groups ( $p>0.05$; Figure 2).

There were no significant differences observed in the context of erythrocyte transfusion, plasma transfusion, blood loss, urine volume between the two groups ( $p>0.05)$. When compared with the SVV 9\% group, the infusion volume in the SVV 14\% group decreased $(p<0.05)$. The difference observed in the amount of norepinephrine was particularly significant between the two groups, with $161.43 \pm 217.40 \mu \mathrm{g}$ in the SVV $9 \%$ group and $910.74 \pm 489.15 \mu \mathrm{g}$ in the SVV $14 \%$ group respectively ( $p$ $<0.001$; Tablell).

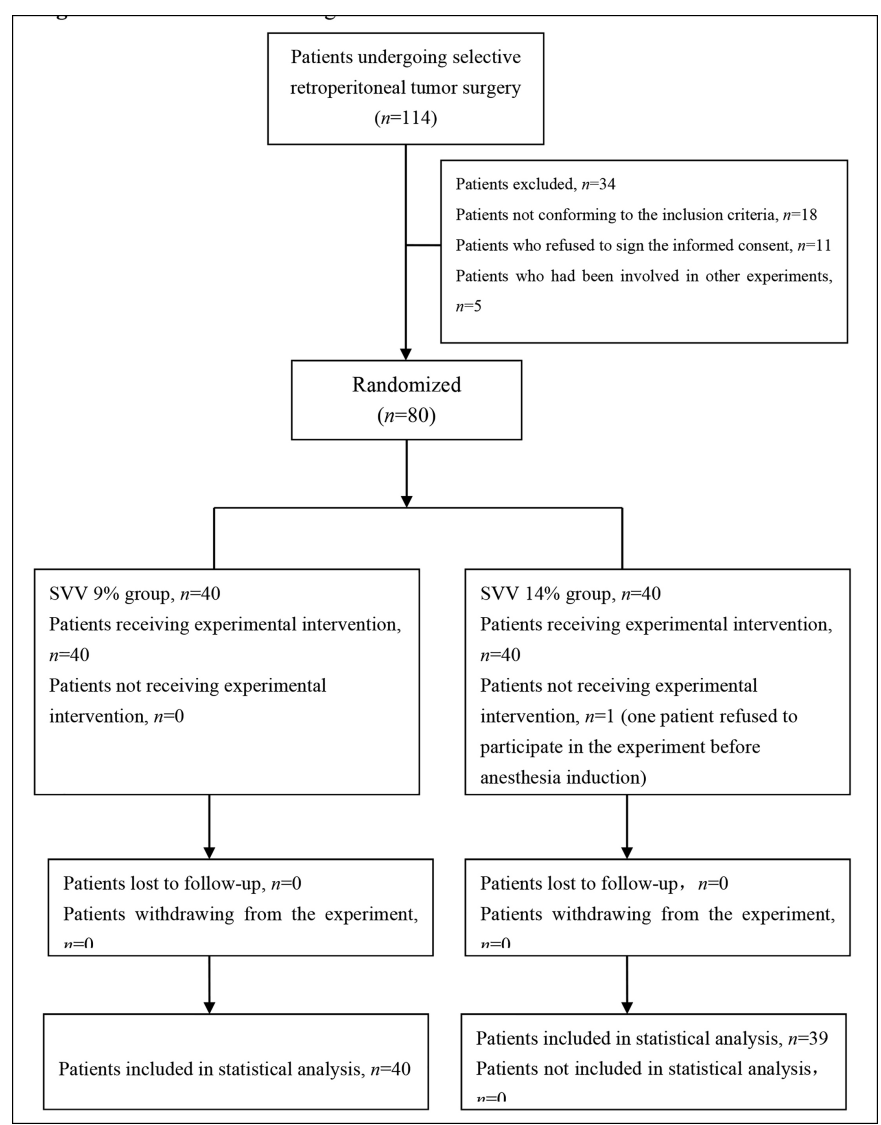

Figure 1: CONSORT flow diagram.

Table l: General data of patients and surgery.

\begin{tabular}{|c|c|c|c|}
\hline & $\begin{array}{c}\text { SVV } 9 \% \text { group } \\
(n=40)\end{array}$ & $\begin{array}{c}\text { SVV } 14 \% \text { group } \\
(n=39)\end{array}$ & $p$ \\
\hline \multicolumn{4}{|l|}{ Patient characteristics } \\
\hline Age (years) (Mean \pm SD) & $49.08 \pm 13.16$ & $47.23 \pm 12.55$ & 0.526 \\
\hline BMI $\left(\mathrm{kg} / \mathrm{m}^{2}\right)($ Mean $\pm \mathrm{SD})$ & $22.05 \pm 2.94$ & $23.32 \pm 3.10$ & 0.067 \\
\hline Males / Females [n (\%)] & $22(55) / 18(45)$ & $19(48.7) / 20(51.3)$ & 0.576 \\
\hline ASA I / II [n (\%)] & $5(12.5) / 35(87.5)$ & $8(20.5) / 31(79.5)$ & 0.337 \\
\hline \multicolumn{4}{|l|}{ Co-morbidities [n (\%)] } \\
\hline Hypertension & $31(77.5)$ & $29(74.4)$ & 0.744 \\
\hline Diabetes & $28(70)$ & $29(74.4)$ & 0.666 \\
\hline \multicolumn{4}{|l|}{ Other (Mean \pm SD) } \\
\hline Operation time (min) & $324.73 \pm 77.72$ & $349.21 \pm 90.00$ & 0.199 \\
\hline
\end{tabular}

The concentrations of SDC- 1, IL- 6 and TNF- $\alpha$ have changed greatly over time $(p<0.001)$ and peaked at T2, while subsequently gradually decreasing post-surgery. Notably, the concentrations at T4 remained higher than those observed at baseline levels. Compared with the SVV 9\% group, serum concentrations of SDC-1, IL- 6 and TNF- $\alpha$ in SVV 14\% group were decreased ( $p=0.028,<0.001,<0.001$, respectively). There was a time-group interaction effect between the time and group factors for IL-6 ( $p<0.001$; Figure 3).

C-SDC- 1 was positively correlated with C-IL-6, C-TNF- $\alpha$ and infusion volume $(r=0.693,0.821,0.820$, respectively; all $p$ $<0.001$ ). In particular, a marked correlation was observed between C-SDC-1 and C-TNF- $\alpha$. Finally, the equation was derived as C-SDC- $1=-3.722+0.216($ C-TNF- $\alpha)+0.031$ (C-IL-6) +0.002 (C-infusion volume). 


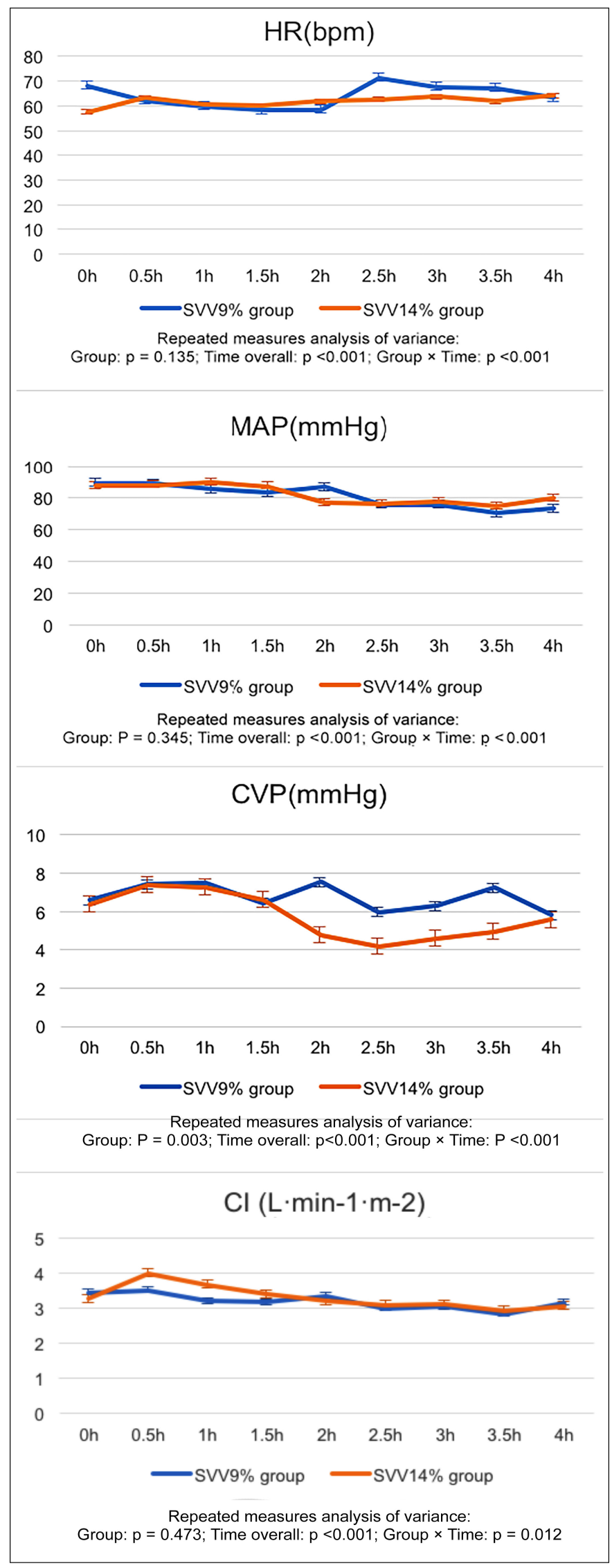

Figure 2: Intraoperative hemodynamics changes.
Table II: Intraoperative fluid infusion and the dosage of vasoactive drugs.

\begin{tabular}{|c|c|c|c|}
\hline & $\begin{array}{c}\text { SVV 9\% group } \\
(n=40)\end{array}$ & $\begin{array}{c}\text { SVV } 14 \% \text { group } \\
(n=39)\end{array}$ & $p$ \\
\hline \multicolumn{4}{|l|}{ Fluid input } \\
\hline $\begin{array}{l}\text { Infusion (ml) } \\
\text { (Mean } \pm \text { SD) }\end{array}$ & $4191.00 \pm 1424.57$ & $\begin{array}{c}3283.46 \pm \\
1144.66\end{array}$ & $0.003^{*}$ \\
\hline $\begin{array}{l}\text { Erythrocyte (U) } \\
\text { [Median (IQR } 25 \text { to 75)] }\end{array}$ & $4(0-4)$ & $4(2-6)$ & 0.800 \\
\hline $\begin{array}{l}\text { Plasma (ml) } \\
\text { [Median (IQR } 25 \text { to 75)] }\end{array}$ & $0(0-400)$ & $0(0-400)$ & 0.863 \\
\hline \multicolumn{4}{|l|}{ Fluid output } \\
\hline $\begin{array}{l}\text { Blood Loss }(\mathrm{ml}) \\
(\text { Mean } \pm \mathrm{SD})\end{array}$ & $1196.5 \pm 796.52$ & $1400 \pm 913.06$ & 0.294 \\
\hline $\begin{array}{l}\text { Urine }(\mathrm{ml}) \\
(\text { Mean } \pm \mathrm{SD})\end{array}$ & $847.50 \pm 427.72$ & $767.95 \pm 287.80$ & 0.334 \\
\hline \multicolumn{4}{|l|}{ Medication (Mean $\pm S D$ ) } \\
\hline $\mathrm{NE}^{\mathrm{a}}(\mu \mathrm{g})$ & $161.43 \pm 217.40$ & $910.74 \pm 689.15$ & $<0.001^{*}$ \\
\hline
\end{tabular}

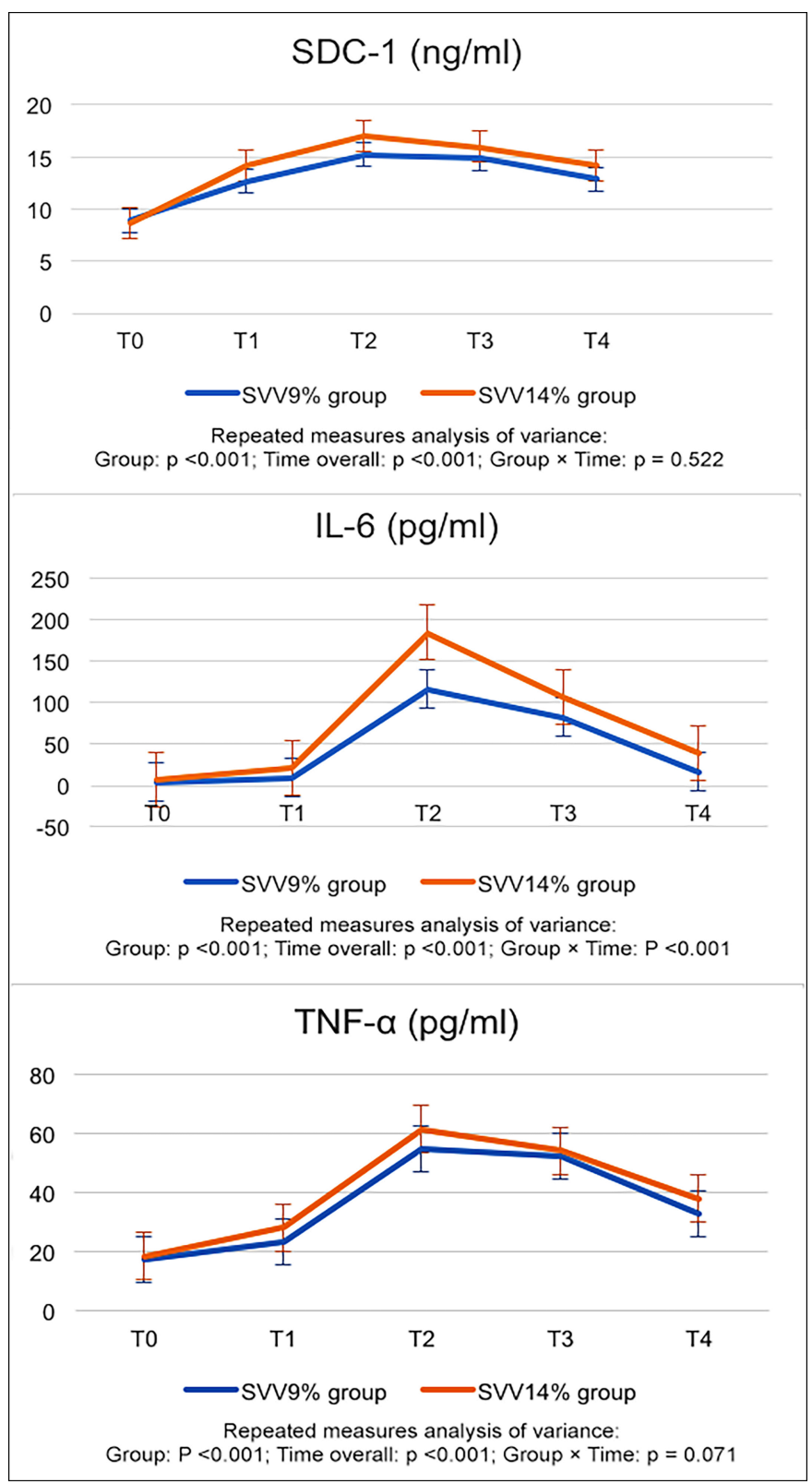

Figure 3. Perioperative changes of SDC-1, IL-6 and TNF- $\alpha$.

*Compared with T0, and at an alpha value of $p<0.05$ in each group. Compared with the SVV $9 \%$ groupat $p<0.05$. 
Table III: Comparison of post-operative conditions between the two groups.

\begin{tabular}{|c|c|c|c|}
\hline & $\begin{array}{c}\text { SVV } 9 \% \text { group } \\
(n=40)\end{array}$ & $\begin{array}{c}\text { SVV } 14 \% \text { group } \\
(n=39)\end{array}$ & $\boldsymbol{P}$ \\
\hline Extubation time (min) & $23.83 \pm 10.66$ & $19.46 \pm 10.82$ & 0.075 \\
\hline Hospital stay $(d)$ & $26.38 \pm 13.06$ & $24.85 \pm 13.22$ & 0.607 \\
\hline \multicolumn{4}{|l|}{ Postoperative complications [ $(\%)]$} \\
\hline \multicolumn{4}{|l|}{ Grade I } \\
\hline Fever $(>37.2)$ & $32(80)$ & $23(59)$ & 0.042 \\
\hline Use of analgesic & $6(15)$ & $5(12.8)$ & 0.780 \\
\hline Use of antiemetic & $10(25)$ & $7(17.9)$ & 0.446 \\
\hline Use of diuretic & $4(10)$ & $0(0)$ & 0.116 \\
\hline \multicolumn{4}{|l|}{ Grade II } \\
\hline Blood transfusion & $18(45)$ & $9(23.1)$ & 0.040 \\
\hline Antibiotic promotion & $5(12.5)$ & $7(17.9)$ & 0.500 \\
\hline \multicolumn{4}{|l|}{ Grade III } \\
\hline $\begin{array}{l}\text { Surgical, endoscopic, or radiological } \\
\text { intervention }\end{array}$ & $2(5)$ & $1(2.6)$ & $>0.999$ \\
\hline \multicolumn{4}{|l|}{ Grade $I V$} \\
\hline Requiring ICU management & $1(2.5)$ & $0(0)$ & $>0.999$ \\
\hline One-year survival rate $[n(\%)]$ & $38(95)$ & $36(92.3)$ & 0.642 \\
\hline
\end{tabular}

Compared with the SVV 9\% group, the incidence of postoperative complications in grade I and grade II in the SVV 14\% group decreased $(p<0.05)$. In addition, more patients in the SVV $9 \%$ group experienced postoperative fever, applied diuretics, and required blood transfusions. There was no significant difference in the extubation duration, length of hospital stay, and the incidence of serious complications between the two groups (all $p$ $>0.05)$. The log-rank test was used to compare survival rates between the two groups, which showed no statistical difference ( $p>0.05$, Table III).

\section{DISCUSSION}

Shedding of the glycocalyx layer on the luminal surface of vascular endothelial cells, an adverse complication of surgery and perioperative hypervolemia, has been a major concern for anaesthesiologist and surgeons in the past few decades. Optimal fluid management is vital for the maintenance and rescue of endothelial glycocalyx , $^{1,3,13,14}$ Reports on the primary monitoring indicators for fluid therapy and titration fluid administration byf anaesthesiologists remain less understood. Previous studies reported that excessive fluid infusion exacerbates glycocalyx shedding as evidenced by elevated SDC-1 concentrations. ${ }^{4,5}$ Belavic et al. showed that $15 \mathrm{ml} / \mathrm{Kg} / \mathrm{hr}$ fluid infusion intraoperatively and $10 \mathrm{ml} / \mathrm{Kg} / \mathrm{hr}$ six hours postoperatively led to higher levels of SDC-1 and hyaluronic acid as compared to $1 \mathrm{ml} / \mathrm{Kg} / \mathrm{hr}$ fluid infusion. ${ }^{5}$ Nevertheless, such empirical fluid management may not precisely determine the effective blood volume in real world. ${ }^{15}$ The present data demonstrated that perioperative glycocalyx shedding products and inflammatory cytokines, namely TNF- $\alpha$ and IL-6, were significantly increased as compared with that prior to surgery. Intriguingly, the SVV 14\% group displayed significantly reduced SDC-1, IL- 6 and TNF- $\alpha$ concentrations compared with the SVV $14 \%$ group, suggesting preserved glycocalyx layer which correlated with improved operational complications. Thus, SVV may be a promising index to guide fluid therapy and setting a target SVV of $14 \%$ could be beneficial for better surgery outcomes.

The timeframe for the glycocalyx layer to repair following surgical damage remains unclear. Potter et al. indicated that it may take 5-7days before the glycocalyx layer restore to its previous thickness. ${ }^{16}$ The present study showed a significant increase in SDC-1 concentrations in a time-dependent manner following surgery. The increased SDC-1 levels were detected at one hour (T1), and plateaued at four hours following skin incision (T2), with a peak concentration of $16.09 \mathrm{ng} / \mathrm{ml}$, which subsequently decreased significantly at 72-hour post-surgery (T4), which was nonetheless still higher than the pre-operative concentrations. A similar trend was found in the concentrations of IL- 6 and TNF$\alpha$. Thus, an intricate fluid management therapy is warranted throughout the relatively long timeframe to ensure reduced damage and faster recovery.

The endothelial glycocalyx layer serves as a vascular barrier that regulates vascular permeability and molecule transport. ${ }^{17,18}$ Inflammatory cytokines, namely TNF-a and IL-6, are also mediators of glycocalyx degradation in animal models and human subjects. ${ }^{19-21}$ Indeed, SDC- 1 is associated with IL-6 and increased mortality in trauma patients. ${ }^{21}$ The present data demonstrate that the concentration pattern of shedding products was comparable with that of inflammatory cytokine, including TNF-a and IL-6 during the perioperative period. Once the glycocalyx layer degraded, the vascular permeability is increased, leading to tissue oedema, augmented leukocyte adhesion, platelet aggregation and dysregulated vasodilation. ${ }^{22}$ In the SVV 9\% group, the higher concentrations of SDC-1 and both inflammatory cytokines are also correlated with higher incidence of postoperative fever, amount of diuretics administration, and blood transfusions required, when compared with SVV 14\% group. However, with regard postoperative 1-year survival rates, there was no difference between both groups, since survival rates of 95 percent were found in the SVV $9 \%$ group and 92.3 percent in the SVV 14\% group. However, incidence of all complications including wound infection, abdominal complications and post-operative hypotension is reduced.

There are several limitations to this study that must be considered. First of all, this is a single-centre pilot study, thus the sample size should be enlarged in future investigations. Secondly, the follow-up period of the postoperative survival status was only pursued for one year after surgery. Thirdly, in this study, the loss of glycocalyx was determined by measuring the plasma concentration of its exfoliated product. If the structure of the ESL layer can be observed by intravital microscopy (IVM), the results will be more intuitive, as this approach has been used in septic mice. ${ }^{23}$

\section{CONCLUSION}

Results supported the efficacy of restrictive fluid therapy during retroperitoneal tumour resection. More importantly, using an SVV-guided goal-directed fluid therapy would reducethe perioperative glycocalyx shedding and ameliorate operational complications. Therefore, this data indicate that SVV $14 \%$ is recommended for preserving the glycocalyx layer in the setting of retroperitoneal tumour resection.

\section{ETHICALAPPROVAL:}

Ethics Committee approval was received for this study from 
the Ethical Committee of Peking University International Hospital, Beijing, China (Chairperson Prof Feng Yu) on 7 July 2016 (Approval No. 2016-029).

\section{PATIENTS' CONSENT:}

Informed consents were obtained from all patients prior to their participation in the study.

\section{CONFLICT OF INTEREST:}

The authors declared no conflict of interest.

\section{AUTHORS' CONTRIBUTION:}

$X W, Y D$ : Involved in experiment design and performed the majority of experiments, analysed results and drafted the paper. ZG: Designed the experiment, supervised and funded the study, and analysed the data.

JG: Data analysis and experiment design.

\section{REFERENCES}

1. Rehm M, Bruegger D, Christ F, Conzen P, Thiel M, Jacob M, et al. Shedding of the endothelial glycocalyx in patients undergoing major vascular surgery with global and regional ischemia. Circulation 2007; 116(17): 1896-906. doi: 10.1161/CIRCULATIONAHA.106.684852.

2. Uchimido R, Schmidt EP, Shapiro NI. The glycocalyx: A novel diagnostic and therapeutic target in sepsis. Crit Care 2019; 23(1):16. doi: 10.1186/s13054-018-2292-6.

3. Astapenko D, Benes J, Pouska J, Lehmann C, Islam S, Cerny V. Endothelial glycocalyx in acute care surgery - what anaesthesiologists need to know for clinical practice. $B M C$ Anesthesiol 2019; 19(1):238.doi: 10.1186/s12871-0190896-2.

4. Nemme J, Hahn RG, Krizhanovskii C, Ntika S, Sabelnikovs O, Vanags I. Minimal shedding of the glycocalyx layer during abdominal hysterectomy. BMC Anesthesiol 2017; 17(1): 107. doi: 10.1186/s12871-017-0391-6.

5. Belavić $M$, Sotošek Tokmadžić $V$, Fišić E, Brozović Krijan A, Strikić $N$, Lončarić Katušin $M$, et al. The effect of various doses of infusion solutions on the endothelial glycocalyx layer in laparoscopic cholecystectomy patients. Minerva Anestesiol 2018; 84(9):1032-1043. doi: 10.23736/S03759393.18.12150-X.

6. Annecke T, Fischer J, Hartmann H, Tschoep J, Rehm M, Conzen $P$, et al. Shedding of the coronary endothelial glycocalyx: Effects of hypoxia/reoxygenation vs. ischaemia/ reperfusion. $\mathrm{Br} J$ Anaesth 2011; 107(5):679-86. doi: 10.1093/bja/aer269.

7. Minto G, Scott MJ, Miller TE. Monitoring needs and goaldirected fluid therapy within an enhanced recovery program. Anesthesiol Clin 2015; 33(1): 35-49. doi: 10.1016/ j.anclin.2014.11.003.

8. Joosten A, Coeckelenbergh S, Delaporte A, Ickx B, Closset J, Roumeguere $\mathrm{T}$, et al. Implementation of closed-loopassisted intra-operative goal-directed fluid therapy during major abdominal surgery: A case-control study with propensity matching. Eur J Anaesthesiol 2018; 35(9): 650-658. doi: 10.1097/EJA.0000000000000827.

9. Fu Q, Mi WD, Zhang H. Stroke volume variation and pleth variability index to predict fluid responsiveness during resection of primary retroperitoneal tumors in Hans Chinese. Biosci Trends 2012; 6(1):38-43. doi: 10.5582/ bst.2012.v6.1.38.

10. Sarris M, Masson JB, Maurin D, Van der Aa LM, Boudinot $P$, Lortat-Jacob $\mathrm{H}$, et al. Inflammatory chemokines direct and restrict leukocyte migration within live tissues as glycanbound gradients. Curr Biol 2012; 22(24):2375-82. doi: 10.1016/j.cub.2012.11.018.

11. Arenal JJ, Bengoechea-Beeby M. Mortality associated with emergency abdominal surgery in the elderly. Can J Surg 2003; 46(2):111-6.

12. Clavien PA, Barkun J, de Oliveira ML, Vauthey JN, Dindo D, Schulick RD, et al. The clavien-dindo classification of surgical complications: five-year experience. Ann Surg 2009; 250(2):187-96. doi: 10.1097/SLA.0b013e3181b1 3ca2.

13. Chappell D, Bruegger D, Potzel J, Jacob M, Brettner F, Vogeser $M$, et al. Hypervolemia increases release of atrial natriuretic peptide and shedding of the endothelial glycocalyx. Crit Care 2014; 18(5): 538. doi: 10.1186/ s13054-014-0538-5

14. Bashandy GM. Implications of recent accumulating knowledge about endothelial glycocalyx on anesthetic management. J Anesth 2015; 29(2):269-78. doi: 10.1007/ s00540-014-1887-6.

15. Nemme J, Krizhanovskii C, Ntika S, Sabelnikovs O, Vanags I, Hahn RG. Hypervolemia does not cause degradation of the endothelial glycocalyx layer during open hysterectomy performed under sevoflurane or propofol anesthesia. Acta Anaesthesiol Scand 2020; 64(4): 538-545. doi: 10.1111/ aas. 13511.

16. Potter DR, Jiang J, Damiano ER. The recovery time course of the endothelial cell glycocalyx in vivo and its implications in vitro. Circ Res 2009; 104(11): 1318-25. doi: 10.1161/ CIRCRESAHA. 108.191585.

17. Alphonsus CS, Rodseth RN. The endothelial glycocalyx: A review of the vascular barrier. Anaesthesia 2014; 69(7): 777-84. doi: 10.1111/anae.12661.

18. Woodcock TE, Woodcock TM. Revised Starling equation and the glycocalyx model of transvascular fluid exchange: An improved paradigm for prescribing intravenous fluid therapy. BrJ Anaesth 2012; 108(3): 384-94. doi: 10.1093/ bja/aer515.

19. Ma X, Liu X, Feng J, Zhang D, Huang L, Li D, et al. Fraxin Alleviates LPS-Induced ARDS by downregulating inflammatory responses and oxidative damages and reducing pulmonary vascular permeability. Inflammation 2019; 42(5):1901-1912. doi: 10.1007/s10753-019-01052-8.

20. Huang L, Zhang X, Ma X, Zhang D, Li D, Feng J, et al. Berberine alleviates endothelial glycocalyx degradation and promotes glycocalyx restoration in LPS-induced ARDS. Int Immunopharmacol 2018; 65:96-107. doi: 10.1016/j.intimp. 2018.10.001.

21. Johansson PI, Stensballe J, Rasmussen LS, Ostrowski SR. A high admission syndecan-1 level, a marker of endothelial glycocalyx degradation, is associated with inflammation, protein $\mathrm{C}$ depletion, fibrinolysis, and increased mortality in trauma patients. Ann Surg 2011; 254(2):194-200. doi: 10.1097/SLA.0b013e318226113d. 
22. Becker BF, Jacob M, Leipert S, Salmon AH, Chappell D. Degradation of the endothelial glycocalyx in clinical settings: Searching for the sheddases. Br J Clin Pharmacol 2015; 80(3): 389-402. doi: 10.1111/bcp.12629.

23. Kataoka H, Ushiyama A, Akimoto Y, Matsubara S, Kawakami
$\mathrm{H}$, lijima T. Structural behavior of the endothelial glycocalyx is associated with pathophysiologic status in septic mice: An integrated approach to analysing the behavior and function of the glycocalyx using both electron and fluorescence intravital microscopy. Anesth Analg 2017; 125(3): 874-883. doi: 10.1213/ANE.0000000000002057. 